Peer-Reviewed Article

ISSN: 2162-3104 Print/ ISSN: 2166-3750 Online

Volume 7, Issue 3 (2017), pp. 748-763

(C) Journal of International Students

http://jistudents.org/

doi: 10.5281/zenodo.570031

\title{
The Lived Experiences of International Students Who's Family Remains At Home
}

\author{
Theresa Harvey \\ Queensland University of Technology, Australia \\ Carolyn Robinson \\ Queensland University of Technology, Australia \\ Anthony Welch \\ Central Queensland University, Australia
}

\begin{abstract}
The significant increase of international students, who leave their family at home, to study abroad, especially in the discipline of nursing, has implications for educational practice. This study's aim was to explore adult international students' experiences of leaving spouse and children - for further education overseas. A descriptive phenomenological approach to inquiry using Colaizzi's (1978) analysis framework revealed four themes: living with emotional turmoil, living in a world of negativity, living with an expectation to cope and driven by personal ambition. The findings identified a hidden world of emotional trauma, psychological stress, and negativity, specifically related to relinquishing their role in the family. The authors conclude that an 'open door policy' that provides individual counselling and timely support for academic and social purposes is necessary.
\end{abstract}

Keywords: International students, connectedness, isolation, acculturation, Internationalization of nursing.

The past 20 years has seen a significant increase in the number of international students entering higher education programs in developed countries. The top four destinations are Australia, United States of America, United Kingdom and France (The Guardian, 2014). In the discipline of nursing, there has been a substantial number of international students who, having left their families at home, travel to a foreign country to undertake further study. Students of nursing continue to be one of the largest groups crossing national borders because of 'pull' factors, such as financial 
incentives, professional development, improved career trajectory, and the opportunity to experience life and work in a different culture (Delucas, 2014; Kingma, 2006; Gardner, 2005).

These mobile and transient groups of students have significant issues to contend with, especially in relation to the emotional support that is required, to live and study in another country successfully, especially when they have left their families at home. Indeed the diversity in cultural and linguistic backgrounds of these students has required learning institutions to set in place strategies to facilitate a smooth and trauma free transition to a different socio-cultural learning environment. Yet, measures undertaken by educational institutions have primarily focused on the development of language competency to meet language requirements for entry to and completion of a program of study (Arkoudis, 2011). What has been omitted in the provision of such services is the recognition of the impact this has on the student who has left their spouse and children for extended periods of time, to undertake further education. Informal discussions with academics, feedback from international students about their educational experience and reflections by the authors of this paper on their experience of working with international students, identified that students in such a situation experience various levels of distress. This is especially evident when they are separated from family, as this impacts on their quality of life and ability to maximize their learning outcomes. This is a significant concern for education providers who host an international student cohort.

The purpose of this paper is to present the findings of a qualitative study about the lived experiences of international adult nursing students who leave their families to undertake undergraduate or post-graduate nursing programs overseas. The study was carried out at an Australian University in the School of Nursing that has a cohort of over 3,000 students.

\section{LITERATURE REVIEW}

The onset and continuation of internationalization in education has significant financial, organizational and social ramifications for the international nursing student (Evans \& Stevenson, 2011; World Bank, 2009). The number of full-fee-paying international students attending education institutions in Australia has increased significantly in the last two decades, from 262,887 in 2002, to 589,860 student enrolments in 2014 (Australian Government, 2015). From the perspective of education institutions the enrolment of international students, apart from providing educational opportunities for students worldwide to advance their career prospects, also provides a financial buffer in an ever competitive and financially starved tertiary sector, particularly in Australia (Arkoudis, 2011). According to Gardner (2005) and Arkoudis (2008), the education sector has undertaken substantial research into the pitfalls confronting both the 
education provider and the overseas student seeking further education. Challenges identified which impact on both the education institution and the international student were, learning and living in a different culture; learning in a foreign university context; lack of interaction between local and international students; learning while developing English language proficiency and academic writing; and becoming familiar with the academic disciplinary discourse (Arkoudis et al, 2013; Arkoudis \& Tran, 2010).

The diversity of cultural backgrounds also creates significant challenges for the education sector. Accepting that culture is defined as a way of life belonging to a designated group of people (Griswold, 2012; Leininger, 2002), and acculturation as a process that individuals undergo in response to a changing cultural context, it can be argued that the acculturation process can generate for the international student, conflict, frustration and personal struggle (Magnusdottir, 2005). On the other hand Brown (2009) identified in her study concerning younger international students, that a prolonged absence from the familiar home environment can have a positive effect for the student. For example, exposure to new cultural norms and socio-cultural values can give the confident and independent student a degree of freedom from cultural and familial expectations, and opportunities for self-discovery and cross-cultural engagement. However, Magnusdottir (2005) also found that there is a thin line between an experience that threatens and one that strengthens the self.

To date, the education sector's response to the needs of international students has primarily been through an academic lens with particular foci on appropriate curriculum adjustment, the development of language competency, and allowance for cultural difference (Boughton, Halliday \& Brown, 2010). However, social support and connecting with people of similar cultural backgrounds has been identified as significant for students' personal well-being (Shakya \& Horsfall, 2000). Indeed, supportive relationships and social support networks are of high interpersonal significance (Shakya \& Horsfall, 2000; Yeh \& Inose, 2003) for people living in a foreign culture.

The mental health of international nursing students has also been identified as an area of concern (Gardner, 2005; Yeh \& Inose, 2003). The experiences that exacerbate this include: loneliness and isolation; alienation and feelings of being different; a lack of understanding by peers about cultural differences; and coping with insensitivity and discrimination; as well as academic and financial challenges, have been found to negatively affect international students' educational experiences. In turn this impacts on their quality of life and determination to continue with their studies (Gardner, 2005; Yeh \& Inose, 2003).

The issues faced by students who study in countries other than their own can include, feeling like an outsider and wanting to be 'let in', adjusting 
to a different work culture (Zhang, 2012; Magnusdottir, 2005), adjusting to different learning styles, and experiencing overwhelming feelings of homesickness (Clerehan et al., 2012). The majority of research undertaken in relation to international students has focused on young adolescents leaving their family, the effects of distance on their developing minds and the experience of a different culture (Clerehan et al., 2012; Brown, 2009; Gardner, 2005). There is a noticeable paucity of Australian and International research that has specifically focused on adult international nursing students' experience of leaving family including children, in their country of origin for a number of years in pursuit of higher education qualifications. This suggests that this population group is not acknowledged as having specific needs and challenges, as a consequence of their special circumstances. Furthermore, the absence of acknowledgement in orientation programs that this population group may have special needs and challenges, only serves to reinforce that such needs and challenges have yet to be considered. In the perceived absence of research about this population this study was undertaken.

\section{RESEARCH METHOD}

A descriptive phenomenological approach to inquiry in the tradition of Edmond Husserl (1859-1938) and using Paul Colaizzi’s (1978) analysis framework were chosen for this study. Descriptive phenomenology is a systematic process of explicating or bringing to awareness human beings' lived experiences of a particular phenomenon as reflected on, and described by them. Lived experience is at the center of phenomenological inquiry the purpose of which is to explicate the fundamental meanings or essences of phenomena experienced in the concrete life-world of people (van Manen, 2014). The term 'life-world' was used by Husserl to describe the world of intersubjective experience occurring in the everyday lives of human beings. The life-world is accessed through the fields of human consciousness and perception in which individuals construct personal meaning of events in making sense of their everyday world. The nexus between lived experience, constructed meaning of lived experience in the process of making one's sense of existence and what it means to be human is at the heart of phenomenology. Assumptions underlying such an approach to inquiry are as follows: humans create social networks; humans can describe retrospective and prospective life events; patterns and themes surface through intense study of phenomena (Parse, 2001, p. 57). The explication of patterns and themes are conduits for accessing the essential nature of a phenomenon through the lens of lived experience. A phenomenological approach to inquiry is consistent with the ideas in health sciences where humanistic understanding is valued (Husserl, 1965; Kim \& Kollak, 2005). 


\section{Participants and Procedures}

Prior to commencement of the study ethics approval was obtained from the University Ethics Committee. An open invitation was then extended to all potential participants through the International Student Centre for the School of Nursing. The invitation contained information about the study including what would be required of participants in the interview process, assurances of confidentiality and anonymity, and the right to withdraw from the study at any time without consequence. Due to the focus of this study, participants were also informed that if they experienced any emotional discomfort during the interview, the interview would be halted and the participant given an opportunity to talk about their discomfort. Participants were also reminded that if they wished to discontinue their involvement in the study their decision would be respected and all information shared by them would not be used unless they were happy for this to occur. A referral process to a professional counsellor located in the student center of the university was set up prior to commencement of the study, in case such a process was required.

Participants who met the inclusion criteria were invited to take part in the study. The inclusion criterion was: being an international student, studying for an undergraduate or post-graduate degree in nursing, and married with both children and spouse remaining in their home country. Ten participants, nine females and one male were recruited. Countries of origin of participants were two from India, two from Indonesia and two from Vietnam, and one each from Brunei, the Philippines, Taiwan and China. All participants were undertaking post-graduate studies in nursing. The time away from home varied between two months and six years. The age of the children that remained at home ranged from one year to late adolescence, with the majority of children between one and five years of age. All participants' children were being cared for by their spouse and the extended family in their home country.

\section{Data collection and Analysis}

The interviews were conducted by two of the authors who are lecturers in the school and have substantive qualitative research experience. They had no personal contact with the participants preceding the recruitment and interview phases of the study. Interviews were arranged at a time convenient to the participants and were held in the researchers' office where privacy was assured. This offered a safe and supportive environment in which participants were able to share their experiences.

Prior to commencement of the interview process, written informed consent was obtained. Interviews were semi-structured, in-depth and conversational in nature. The opening question framed as a request was: 
"Please describe your experiences of leaving your children and spouse at home in order to undertake studies in Australia?” Additional questions asked during the interview process for clarification and elaboration were: "can you give me an example about what you have just described?" "Tell me more about that and, what were you feeling and thinking at that time?" Each interview took approximately one hour.

During the interviews three participants displayed emotional discomfort at which time the researcher halted the interview and provided the participants the opportunity to discuss any concerns. The participants were also offered the opportunity to discontinue the interview and to access the counselling service. All three participants expressed a desire to continue and therefore the interviews resumed. The option of counselling was refused by all three participants. Each interview was digitally recorded and subsequently transcribed by a research assistant in preparation for analysis.

Analysis of the transcripts using Colaizzi's (1978) approach commenced with all the authors repeatedly reading each transcription of interview while listening to the digital recordings in order to gain an overall feeling of participants' experiences. This process was followed by a review of each transcript of interview by each of the authors to identify significant statements in the form of phrases and sentences. Once this stage had been completed the three authors came together to share their individual analyses and to work toward a consensus of significant statements, aggregation of categories and the development of theme clusters. The researchers moved back and forth between the participants' transcripts, the significant statements explicated from the transcripts reflecting the participants' experiences and the aggregated categories to ensure accuracy in the process of interpretation of participant statements. At the conclusion of this process the aggregated categories were then further aggregated resulting in the development of four themes.

Data saturation began to occur at participant eight, however to ensure this was the case, transcripts of participants' nine and ten were analyzed to confirm data saturation had been achieved, and this was confirmed. The final number of ten participants for this study is consistent with the recommendation for conducting phenomenological inquiry, that is, between six to ten participants is considered an adequate sample size given the large amount of rich experiential data collected (Morse, 2000; Denzin \& Lincoln, 1994).

Lincoln and Guba's (1985) framework for ensuring rigor in qualitative inquiry was applied to this study. This involved four principles: credibility, dependability, transferability and conformability. Credibility was achieved by ensuring accuracy in describing the participants' experiences. This involved returning the analysis of transcripts to participants for validation. Dependability, which is concerned with the integrity of the 
researcher to be true to the presentation of findings was achieved by each of the authors undertaking an audit of each other's process of analysis by working back from the findings to the original transcripts of interview. Transferability is where the findings are viewed by others in similar situations as having meaning for them. This principle was achieved by providing an overview of the findings to other international students in similar circumstances to the participants. There was general agreement that their own experiences were similar to the findings of this study. Trustworthiness of the study was achieved by meeting the requirements of the other three principles of research rigor.

\section{FINDINGS}

Findings of the study revealed four major themes that were linked to the participant's struggles in which they had relinquished their main role within their immediate family, and their attempt to adapt to a life of educational and socio-cultural differences. The four themes: living with emotional turmoil; living in a world of negativity; living with an expectation to cope, and driven by personal ambition revealed a world of hidden emotional trauma and psychological stress experienced by participants.

\section{Living with emotional turmoil}

The first theme to emerge was living with emotional turmoil. In sharing her experiences of emotional turmoil one participant articulated, "[I] feel very alone, isolated and homesick because we are used to living in big families ... [my daughter] is very close to me ... it was hard for me [to leave her]... I am emotionally torn between staying and returning home”. Another recalled struggling to overcome her feelings of isolation, "You never really overcome the feeling of being alone and isolated when your family is not with you. At times it becomes overwhelming leaving you in an emotional state of turmoil. When this happens you just feel like leaving everything and returning home, but you know you can't do that. You just have to live with all those conflicting emotions".

A number of the participants wrestled with the pragmatic need not to be with family during their studies as described by one, "actually being alone which I sometimes feel has its pros and cons, my focus has to be on my study and only sometimes for my kids, but I continually miss them ... the saddest thing is being away from them. Sometimes when I attempt to study I can't, as thoughts of my family and being away from them prevent me from concentrating, which I need to do, and I end up in tears. It is like being caught in a no win situation”.

For some the emotional turmoil surfaced in the form of conflict and guilt as described by one of the participants: "I sometimes feel [leaving 
family] was a mistake ... When this happens I feel extremely guilty. I know I shouldn't, but the guilt just sits there as if I have done something wrong. I am often torn between thinking about my own needs and leaving my family to achieve them, both of which make me feel guilty". Another stated the experience was one of anxious uncertainty, "sometimes I feel very anxious and uncertain about whether my decision was wrong or right". While another spoke of her feelings of disquiet as she struggled to come to terms with handing over family responsibilities: "Having to leave my family to study has not been an easy thing. You feel deep down that it is the right thing, but also feel guilty for handing over the daily responsibility to family when they have their own responsibilities to carry out”.

To cope with their emotional turmoil all participants commented in one way or another that regular communication was paramount in being assured that all was well at home and to keep their thoughts and emotions under control. As one explained "I talk almost every day [to my daughter] and this gives me comfort...then I can focus on my study. I'm trying hard not to let the emotions get in the way of my completing my studies”. Living with emotional turmoil was accompanied by living in a world of negativity.

\section{Living in a world of negativity}

Feelings of emotional turmoil were exacerbated by underlying feelings of living in a world of negativity, the second theme identified in this study. One participant said: "I am constantly thinking about what can go wrong to prevent me from finishing my studies. Negative thoughts about being away from home, not being successful with my studies and getting sick are often there. At times such thoughts make me depressed and anxious. I try to keep busy, to ignore such feelings and thoughts, to stop being pulled down by negative thoughts but it is difficult".

Living in a world of negativity was further identified by another who explained that even when she was home with her family during the period of data collection for her dissertation she was: "unable to enjoy their company [family], as I still had to focus on my research. I often question why I am doing this to the family and myself. Life at the moment is all negative".

Another who felt the need to eventually bring her young son to Australia to alleviate her feelings of guilt at not being able to care for him, found herself in an ever increasing spiral of unrelenting negativity: "as a student here, it is kind of difficult, everything at times seems to be a burden - the constant pressure of study, and now caring for my child, trying to make friends and just wanting to feel accepted by others. Not being able to care for my child as I would at home amongst all the other struggles is so depressing at times... I can't see that this will ever change while I am here 
studying”. Living in a world of negativity was exacerbated by feeling that they were expected to cope.

\section{Living with an expectation to cope}

For all the participants, living with uncertainty about making the right decision to study abroad, feeling guilty for leaving family to study, and trying to cope with anxiety and the presence of negative thoughts that impacted on their ability to study was an ongoing struggle. However, seeking help and support from family or health professionals was described by one participant as “not an option as I don't want to cause any distress or worry to them ... If I shared my thoughts and feelings it would only be another source of worry". This revealed the third theme of living with an expectation to cope. Another participant spoke of the difficulty in not being able to confide in others whether that be family or seeking some form of professional help, "that is not what we do. We are expected to cope despite difficulties we encounter so we are expected to keep such thoughts and feelings inside... talking to a health professional is also not something we do easily. It is better to keep such things to yourself”. This was also identified by another participant who spoke of feeling depressed but not willing to seek help because of the fear of being seen as a failure: "If I let people know that I am not coping I will be looked down on. It is a sign of weakness... although at times I feel I need some help there is nothing I can do about it”. However, in the face of such difficulties all participants continued to forge ahead with their studies driven by personal ambition.

\section{Driven by personal ambition}

All participants in this research continued with their studies, driven by personal ambition which was identified as theme four. This was despite experiences of emotional turmoil, negativity, mounting perceived disconnection and expectations that they needed to cope with these difficulties. They recognized that this period of separation from family was something that they needed to do, for example as described by one participant: "It was very difficult to make this decision that I am going to pursue my studies, because my daughter is really small and because I want to see her growing up, but this is something I have to do for her and me ..." This was expanded further by another participant who said: "I do meditation every day to keep me in balance, to keep me relaxed and to refresh my mind and concentrate on my studies, ... to achieve my goals, ... it's very hard. Sometimes I feel mentally upset, I want to touch her [my daughter] and hug her...I miss her, but my studies are also important. If I am successful with my studies I can make a better life for her". 
This sentiment was also articulated by another participant who said: "it's very difficult [to study overseas] but the results of doing so are worth it. It is something I have to do". Another example was when one participant felt that staying focused on her goal was the best way forward: "I really feel sorry for her [eldest daughter 20 years old] because she has taken on my role in the home...I feel terrible but I try to ignore it because my goal is to be successful and be able to provide financially for the family". Similar sentiments were expressed by another who stated: "I feel sad because I miss them [the family] but I also realize my responsibility is to keep focused, then I'll finish on time and that will be good for me and good for them".

The findings from this research have identified that participants had deep seated emotional turmoil that was further impacted on by negativity and the pressure to cope and succeed. These issues appear to be hidden consequences that are not readily acknowledged by educational providers.

\section{DISCUSSION}

There is considerable research that explores the experiences of international students undertaking studies abroad, with the majority of these looking at young adolescents. A small number of studies explored international students' experiences of leaving spouse and children at home to undertake studies outside their country of origin. The findings in the literature were in many respects similar to some of the issues identified in our study. For example Yeh and Inose's (2003) study of predictors of a-cultural stress in students conducted in the North East of the United States of America, identified that some students experience greater difficulty on an emotional level when apart from their family and friends. Similarly to these findings, Zhang's (2012) study of acculturative stress among Chinese international students, found that loss of social support has a significant influence on the psychological well-being of international students, who often experience difficulty establishing new social supportive relationships. Walters' (2006) narrative study of 16 nurses from South Asia, about their journey through interview, selection, arrival, registration and integration within the Australian nursing system, found that educational providers tended to advise international nursing students who have a spouse and/or child still living in their homeland, to leave their family at home while they complete their studies. The rationale for such advice was to suggest the presence of family can be a source of distraction from studies, as well as issues with accommodation, finding employment for the spouse if required and, accessing childcare if needed. Gardner's (2005) research which explored barriers influencing the success of ethnic minority students in nursing programs conducted in California, identified pitfalls experienced by international students which included: language, learning in a foreign university, academic disciplinary discourse, being an outsider and needing 
to be 'let in' and overcoming work culture challenges, as the major concerns.

In our study it was found that all ten participants identified these same concerns of being exposed to a different academic way of life, learning styles and a mismatch of expectations. What was most significant in our study was the overriding and difficult issue they constantly faced of leaving behind spouse and children. These findings are in contrast to younger, confident students as identified in Brown's (2009) study in the South of England, where students who left their familiar home environments, were afforded the opportunity of freedom from cultural and family expectations, allowing them opportunities for personal self-discovery. Conversely, Markus and Kitayama (1991) and Zhang (2012) reported being separated from important others such as family who have played a central role in the development of the person's self-image, can lead to a decrease in the person's self-identity, resulting in significant distress. While relationships are important to all students, for the participants in our study, being connected with their loved ones, especially their children at home was identified as an essential aspect of their self-identity, values, and ways of interacting as individuals.

Yeh and Inose (2003) also identified a lack of English fluency, social support, satisfaction, and social connectedness were predictors of acculturative stress. The findings of their study indicated international students are potentially at risk of developing mental health problems as a result of their circumstances. However, no further description of what this could mean was provided. In our study all the participants identified specific issues relating to their mental health, including their daily struggle with sadness, loneliness, lack of perceived acceptance, homesickness and guilt which were directly connected to leaving family. They also expressed feelings of loss at relinquishing their family roles for a period of time, which included the daily responsibility of caring for their family, in order to focus solely on themselves and their studies. Interestingly, an unexpected outcome from our study revealed that all participants felt relief at being able to express their pent up feelings during their interview, the intensity of which they had not recognized. The highlighting of these emotional experiences, would suggest that education providers should acknowledge and address these openly, especially in orientation programs. This is because students themselves are unaware of the impact of their situation, prior to commencing their studies abroad.

Success for the participants was multi-layered, involving achieving outcomes for themselves personally, for their country of origin on their return, and most importantly in the long term for their family. Securing a financial future that afforded their family a better education and quality of life was the overall objective. This was summed up by a participant who 
described their experience of leaving family and home to study abroad as "a necessary sacrifice". Emerging from the literature and the analysis of our research was the participants' struggle with being separated from family and the need to belong, made more intense by the presence of loneliness and homesickness which remained hidden from family and friends. The four core themes identified in our study further impacted on each participant's sense of self and feelings of isolation. In an attempt to combat their sense of isolation from family, they employed a range of strategies including: regular communication with family using Skype and email; engaging in periods of personal prayer; and taking time out to meditate. Some also avoided or minimized socializing with others when and where possible, in order to lessen feelings of guilt associated with being away from their spouse and children, allowing them to focus on their studies.

The four themes identified were not separate disconnected entities but interrelated, providing a conceptual understanding of the participants' experiences and motivation to complete their course of study (See diagram 1). The experiences of emotional turmoil involved having to contend with conflicting emotions about making the right choice between family and study abroad. At times, they all expressed being engulfed in negative thoughts of guilt, loneliness, self-questioning and ongoing worry, where they internalized these overwhelming feelings so they did not burden others. However in the face of emotional turmoil, negativity and being expected to cope, participants' personal ambition to succeed was the driving force to complete their studies. The contribution to the development of new knowledge explicated in our study relates not only to the four overarching themes, but also to the specific content of each theme and their interrelationships. These findings need to be acknowledged by education providers, to prevent negative outcomes.

\section{Diagram 1 - Interrelated categories leading to achievement}






\section{CONCLUSIONS \& SIGNIFICANCE}

Challenges confronting international students who study abroad have been found through this study to extend beyond language and academic concerns to touch the very fabric of their daily lives. The experiences of separation from family, loneliness, homesickness, feelings of guilt and anxiety about whether they had made the right decision and reluctance to share their difficulties with family weighed heavily. This was compounded by their reluctance to seek assistance from health professionals for fear they would be perceived as not coping, underpinned daily living for this group of students. However, their resolve to complete their course of study in the face of mounting difficulties was driven by personal ambition and determination to succeed.

Educational institutions have well established procedures and protocols to address the linguistic, cultural and educational needs of international students to facilitate and enhance their educational experience. The university at which this study was undertaken has established procedures for meeting the academic and socio-cultural needs of international students which include: orientation programs specifically designed to provide information about the Australian culture and social mores, the university as a learning institution; academic requirements and protocols, and academic support services. Students are also informed about university events including social, cultural and sporting activities that are aimed at assisting international students to develop supportive networks during their time of study.

Education and information sessions have also been implemented for academic and administration staff aimed at 'awareness development' of the needs of international students, from both academic and personal perspectives, with a clear focus on a positive holistic educational experience. However, one significant area that has yet to be addressed by education providers is the hidden world of international students as identified in this study. The apparent lack of acknowledgment and attention to the needs of international students who leave their spouse and children to study abroad has the potential to place these students psychologically and emotionally at risk. It is therefore incumbent on education providers to start thinking about ways by which such issues raised in this study can be addressed.

The need for education providers to find 'a bridge' to working with students caught in such circumstances is now required. What form this bridge should take has yet to be established however, a number of initiatives could include: The identification of the potential risks for this student population prior to commencement of their program of study; utilizing existing support infrastructures within the university to develop a collaborative approach including counselling services, student support 
services and academics working with this population, to identify potential and emerging patterns of not coping; making provision for students to engage in open dialogue which may involve an 'open door policy' for timely support and counselling.

The findings of this study provide a different conceptual lens from which to view the daily lived world of this population group to previously identified challenges experienced by international students. The four themes (living in a world of negativity; living with emotional turmoil; living with expectations to cope: driven by personal ambition) draw attention to the hidden difficulties faced by this population of international students. The absence of research in this area and the significance of the findings of this study suggest further research is necessary on the cross-cultural adjustment for this population group.

\section{REFERENCES}

Arkoudis, S., Watty, K., Baik, C., Yu, X., Borland, H., Chang, S., Lang, I., Lang, J., \& Pearce, A. (2013). Finding common ground: enhancing interaction between domestic and international students in higher education. Teaching in Higher Education,18(3), 222-235.

Arkoudis, S. (2011). Teaching international students: Strategies to enhance learning. University of Melbourne, Australia: Centre for the Study of Higher Education. Retrieved from http://www.cshe.unimelb.edu.au/pdfs/international.pdf

Arkoudis, S., Tran, L. (2010). Writing Blah, Blah, Blah: Lecturers’ Approaches and Challenges in Supporting International Students. International Journal of Teaching and Learning in Higher Education, 22(2), 160-178.

Arkoudis, S. (2008). Taking the role of English language seriously in higher education: Why is it so difficult? Retrieved from http://www.cshe.unimelb.edu.au/resources_teach/teaching_in_practice/docs/in ternational.pdf

Australian Government. (2015). Department of Education and Training. International student enrolments in Australia 1994 - 2014. Retrieved from https://internationaleducation.gov.au/research/International-Student-

Data/Pages/InternationalStudentData2014.aspx

Brown, L. (2009). The transformative power of the international sojourn: An ethnographic study of the international student experience. Annals of Tourism Research, 36(3), 502-521.

Boughton, M., Halliday, L., \& Brown, L. (2010). A tailored program of support for culturally and linguistically diverse (CALD) nursing students in a graduate entry masters of nursing course: A qualitative evaluation of outcomes. Nurse Education in Practice Journal, 10, 355-360.

Clerehan, R., McCall, L., McKenna, L., Alshahrani, K. (2012). Saudi Arabian nurses' experiences of studying Masters degrees in Australia. International Nursing Review, 59(2), 215-221. 
Colaizzi, P. (1978). Psychological research as the phenomenologist views it. In: Valle, R.S., \& King, M. (Eds), Existential Phenomenological Alternatives for Psychology, (48-71). New York, NY: Oxford University Press.

Delucas, A.C. (2014). Foreign Nurse Recruitment: Global risks. Nursing Ethics. 21(1), $76-85$.

Denzin, N.K., \& Lincoln, Y.S. (1994). Handbook of qualitative research. Thousand Oaks, CA: Sage.

Evans, C. \& Stevenson, K. (2011). The experience of international nursing students studying for a $\mathrm{PhD}$ in the U.K: A qualitative study. BMC Nursing 10(11), 113.

Gardner, J. (2005). Barriers Influencing the Success of Racial and Ethnic Minority Students in Nursing Programs. Journal of Transcultural Nursing, 16(2), 155162.

Griswold, W. (2012). Culture and Societies in a Changing World. Thousand Oaks, CA: Pine Forge Press.

Husserl, E. (1965). "Philosophy as a Rigorous Science" in Phenomenology and the Crisis of Philosophy. Harper \& Row: NY. 71-147

Kim, H., \& Kollak, I. (2005). Nursing Theories: Conceptual and Philosophical Foundations. $\left(2^{\text {nd }}\right.$ Ed) Springer Publishing Co: University of Michigan

Kingma, M. (2006). Nurses on the mover: Migration and the Global Health Care Economy. ILR Press/Cornell University Press, Ithaca, NY.

Leininger, M. (2002). Transcultural nursing: Concepts, theories and practices. $\left(3^{\text {rd }}\right.$ Ed) New York: McGraw Hill Professional.

Lincoln, Y.S. \& Guba, EG. (1985). Naturalistic Inquiry. Newbury Park, CA: Sage Publications.

Magnusdottir, H. (2005). Overcoming strangeness and communication barriers: a phenomenological study of becoming a foreign nurse. International Nursing Review, 52(4), 263-267.

Markus, H.R. \& Kitayama, S. (1991). Culture and the self: Implications for cognition, emotion, and motivation. Psychological Review, 98, 224-253.

Morse, J.M. (2000). Determining sample size. Qualitative Health Research, 10(1), 3-5.

Parse, R. R. (2001). Qualitative inquiry: The path of sciencing. Jones and Bartlett Publishers: Canada

Shakya, A., \& Horsfall, J. (2000). ESL undergraduate nursing students in Australia: Some experiences. Nursing and Health Sciences, 2,169.

The Guardian (2014). Top 20 countries for international students, Retrieved from http://www.theguardian.com/higher-education-network/blog/2014/jul/17/top20-countries-international-students

Van Manen, M. (2014). Phenomenology of Practice. USA: Left Coast Press, Inc.

Walters, H. (2006). The experiences, challenges and rewards of nurses from South East Asia in the process of entering the Australian nursing system. Australian Journal of Advanced Nursing, 25(3), 104.

World Bank. (2009). The nurse labor and education markets in the Englishspeaking CARICOM: issues and options for reform. Washington, DC: World Bank. 
Yeh, C., \& Inose, M. (2003). International students reported English fluency, social support and satisfaction, and social connectedness as predictors of a-cultural stress. Counselling Psychology Quarterly, 16(1), 16.

Zhang, Y. (2012). An Examination of Acculturative Stress, Perceived Social Support and Depression among Chinese International Students. Retrieved from http://surface.syr.edu/cfs_thesis/3

THERESA HARVEY is an Academic and currently is serving as the Director of Clinical Partnerships in the School of Nursing, Queensland University of Technology. Her research interests include clinical education for both students and health professionals, and supporting culturally and linguistically diverse students to transition in to the Australian education and health care environment. Email: tm.harvey@qut.edu.au

CAROLYN ROBINSON is a Sessional Academic in the School of Nursing Queensland University of Technology. She has focused her career on designing and presenting multidisciplinary workshops to meet the specific needs of both students and health professionals in relation to clinical practice and supervision of people from culturally and linguistically diverse backgrounds. Email: c.robinson@qut.edu.au

ANTHONY WELCH is Associate Professor Mental Health Nursing and, Assistant Dean, Community Engagement at Central Queensland University, Noosa. His research expertise is in qualitative and mixed methods modes of inquiry. His particular foci of research is on mental health, resilience, suicide prevention, men’s health, spirituality and recovery. Email: a.welch@cqu.edu.au

Manuscript submitted: May 12, 2016

Manuscript revised: November 30, 2016 Accepted for publication: January 2, 2017 